\title{
An analytic model employing an elliptical surface area to determine the gaseous thermal conductance of uncooled $\mathrm{VO}_{\mathrm{x}}$ microbolometers
}

\author{
J. Schoeman ${ }^{\mathrm{a}, *}$, M. du Plessis ${ }^{\mathrm{a}}$ \\ ${ }^{a}$ Carl and Emily Fuchs Institute for Microelectronics, University of Pretoria, Lynnwood Rd, Pretoria, South Africa
}

\begin{abstract}
This work presents a detailed overview of the analytic methods for calculating the beam and gaseous thermal conductance components associated with uncooled $\mathrm{VO}_{\mathrm{x}}$ microbolometers. The conventional method to calculate the gaseous component relies on the assumption that the entire plate is maintained at a uniform temperature, thus the surface area of the plate is used for the calculation. We have observed using an industry leading multiphysics simulator that this assumption is not strictly true for $\mathrm{VO}_{\mathrm{x}}$ microbolometers as the conduction pattern exhibits an elliptical shape. Based on this, we have developed and propose an analytic method that employs an elliptical surface area scaled appropriately with the device dimensions to obtain an estimate of the average temperature conduction pattern. Prototype devices were manufactured and experimentally characterised. The devices exhibit thermal conduction characteristics comparable to those in literature and industry, and we could achieve $0.5 \mu \mathrm{W} / \mathrm{K}$ under vacuum conditions and $15 \mu \mathrm{W} / \mathrm{K}$ at atmospheric pressure with a TCR of $-1 \% / \mathrm{K}$. However, both simulated and experimental result sets of the gaseous thermal conductance exhibit large deviations from the conventional analytic method, on average approximately $40 \%$. The proposed method reduces this average error significantly to less than $10 \%$ when compared to the simulated results.
\end{abstract}

Keywords: elliptical area model, thermal conductance, microbolometers, MEMS

\section{Introduction}

Since the commercialisation of the MEMS based uncooled microbolometer, most notably due to the advances in micromachining leading to high yield and low cost manufacturing, a vast range of applications for thermal imaging has seen the light. These applications have penetrated the industrial, military and consumer markets, indicating their diversity and the extensive marketing opportunities they present, ranging from examples in security, surveillance, and vehicle, pedestrian and wildlife monitoring systems [1, 2, 3, 4, 5, 6]. Futhermore, these sensors have contributed significantly to thermography for medical applications, including inflammation detecting and classification of and detection of certain malignancies [7, 8, 9, 10, 11]. However, microbolometers are not only limited to thermal imaging, but have also been adopted as pressure sensors [12].

The rise in the popularity of uncooled microbolometer focal point arrays (FPAs) using vanadium oxide $\left(\mathrm{VO}_{\mathrm{x}}\right)$ as a detector material has also encouraged improvements in the analytic modelling of the thermoelectric device behaviour. Some of the recent advances include analytic models that not only considers the dimensions of the support beams as conventionally done, but also the influence of the device plate that causes a radial heat flow component and constriction resistance resulting from a discontinuity in width when the beam and plate are connected

\footnotetext{
* Corresponding author

Email addresses: johan.schoemandeng.up.ac.za (J. Schoeman), monuko.duplessis@up.ac.za (M. du Plessis)
}

[13]. This model is limited to the beam thermal conductance that dominates device behaviour under vacuum conditions.

Although $\mathrm{VO}_{\mathrm{x}}$ microbolometers are mostly used as detectors under vacuum conditions to achieve high sensitivity, drawbacks exist when capturing scenes with high thermal dynamic range or high temperature regions like explosions, fire, fuselage and the sun [14, 15]. The output of these sensors saturate and take a long time to recover. Furthermore, vanadium oxide is thermochromic, therefore its absorption and resistance properties change if the detector temperature exceeds about $68^{\circ} \mathrm{C}$ as a semiconductor-metal phase transition occurs [16]. Devices operated at atmospheric pressure are better suited for high temperature operation due to the inclusion of an ambient or gaseous thermal conductance component of the microbolometer. This is conventionally modelled as a simple function of plate geometry, which we will show to be only a crude approximation based on simulation and experimental results. Some improvements to device modelling at atmospheric pressure do exist. The first improvement resulted from employing microbolometers as microPirani pressure sensors to monitor the quality of the vacuum within the packing, and incorporates the effect of the operating pressure into the model. They rely on the fact that the mechanism for determining air pressure is highly linked to the gaseous thermal conduction component. [12, 17, 18]. It has also been proposed for metal thin-film microbolometers that an improved surface area is achieved when including a portion of the support beam [19], as well as the side-wall thermal conduction associated with the plate and additional beam area [20]. However, it became clear to us that none of these methods apply particularly 
well to specifically $\mathrm{VO}_{\mathrm{x}}$ based devices operated at atmospheric pressure and that no accurate analytic method for thermal conductance exists to model this operating condition.

The main contribution of this paper is to address the current shortcoming in literature for the accurate analytic modelling of the gaseous thermal conductance component of uncooled $\mathrm{VO}_{\mathrm{x}}$ microbolometers under atmospheric pressure conditions by introducing a method based on an elliptical surface area. It should be highlighted that the presented method can also be applied to microbolometers with any detector material where the resistivity is significantly higher than that of the metal used on the support beam to connect to the detector material, but only at atmospheric pressure. The technique does not apply to vacuum conditions where a constriction type model offers better results [13], nor to metal type microbolometers like thin-film Ti based devices, where an extended surface area method including a portion of the support beams provide the best results [20]. In the sections to follow, the authors will present the theoretical background and proposed improvement to the conventional analytic model, the device designs manufactured for this study, the approaches followed for both the simulation and experimental methods, the results and discussion and finally the conclusion and acknowledgements.

\section{Theory and modelling of microbolometers}

\subsection{Operating principles of microbolometers}

The detector consists of a multilayer structure referred to as the plate or membrane suspended in a bridge like manner over a cavity, connected to a substrate by means of thin connectors typically referred to as support beams or legs. The different layers play a role in structural support, electrical isolation, insulation and detection. This detector volume can store absorbed energy and acts like a thermal capacitance, $H$. Therefore, any incident radiation will be absorbed by the thermal capacitor and will heat up the suspended plate and sensor material by means of electromagnetic (thermal) radiation. However, since a temperature gradient exists between the detector membrane at temperature $T_{m}$ and the substrate at a fixed temperature, $T_{s u b}$ through the legs and atmosphere, heat flow will occur by means of the conduction principle. This is modelled by the total or effective thermal conductance, $G_{e f f}$, that consists of a number of thermal conductive paths as described later. The operating principles of a generic thermal detector is illustrated in Fig. 1 .

The heatflow model of a microbolometer can be stated mathematically as [21, 22]

$$
\begin{array}{r}
H \frac{d T_{m}}{d t}+\underbrace{G_{a t m}\left(T_{m}-T_{a}\right)+G_{l e g}\left(T_{m}-T_{\text {sub }}\right)+P_{\text {rad }}}_{G_{\text {eff }} \Delta T} \\
=\underbrace{P_{I V}}_{P_{e}}+\underbrace{\varepsilon_{I R} P_{I R}+\varepsilon_{s u b} P_{s u b}}_{Q}
\end{array}
$$

where

- $H$ is the thermal capacity of the detector with unit $\mathrm{J} / \mathrm{K}$,

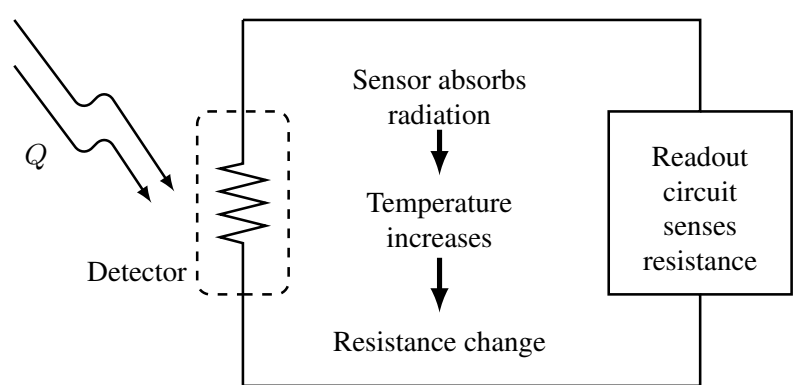

(a) The principles of operation of a generic thermal detector

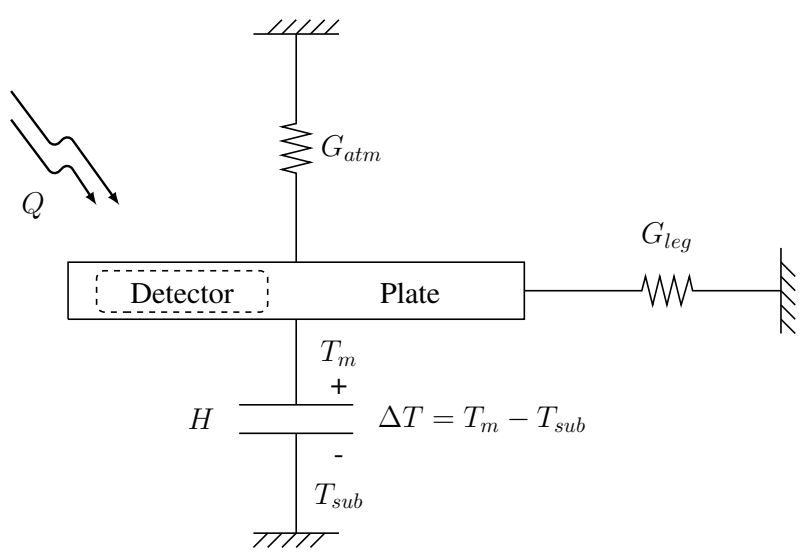

(b) The the equivalent heat flow schematic of a generic thermal detector

Figure 1: The operating principles and the thermal model for a generic thermal detector.

- $G_{l e g}$ is the thermal conductance of the detector to the substrate via the supporting legs in $\mathrm{W} / \mathrm{K}$ (often $\mu \mathrm{W} / \mathrm{K}$ ), analogous to the thermal resistance that results in losses in heat,

- $G_{a t m}$ is the thermal conductance of the detector through the atmosphere to the substrate with unit $\mathrm{W} / \mathrm{K}$ (often $\mu \mathrm{W} / \mathrm{K})$,

- $T_{m}$ is the temperature of the membrane in $\mathrm{K}$,

- $T_{a}$ is the ambient temperature surrounding the membrane in $\mathrm{K}$,

- $T_{\text {sub }}$ is the temperature of the substrate in $\mathrm{K}$,

- $P_{\text {rad }}$ is the power radiated from the thermal detector into its surroundings in $\mathrm{W}$,

- $P_{I V}$ is the electrical power $\left(P_{e}\right)$ applied to bias the detector in $\mathrm{W}$,

- $\varepsilon P_{I R}$ is the portion $\left(\varepsilon_{I R}\right)$ of incident infrared radiation absorbed by the detector in $\mathrm{W}$,

- $\varepsilon P_{s u b}$ is the portion $\left(\varepsilon_{s u b}\right)$ of radiated power absorbed by the detector that is emitted from the substrate when it is not at zero Kelvin, with unit W, 
- $Q$ is the total absorbed radiative power from all the radiating sources in W.

$G_{\text {rad }}$ is defined as the change in radiation power observed with small changes in temperature and it is significantly smaller than $G_{l e g}$ under all normal operating conditions when considering the thermal losses, $G_{e f f} \Delta T$, in typical microbolometer designs. Therefore, it is often assumed to have negligible effect and omitted where it simplifies the analytic analysis. Further to this, the main thermal conduction occurs from the membrane through the atmosphere to the substrate if the device is not packaged in an evacuated chamber, therefore $G_{a t m}>G_{l e g}>>$ $G_{\text {rad }}$. A final simplification related to the thermal conduction model can be made when considering the substrate and ambient temperatures, which can be assumed to be the same for uncooled devices with a large substrate.

As briefly mentioned earlier, $G_{\text {eff } f}$ consists of a number of components [17], one of which is a thermal conductance component attributed to heat loss between the suspended membrane and the substrate via the support beams, referred to as the beam or leg thermal conductance, $G_{l e g}$ and is given by [13, 23]

$$
G_{l e g}=N \sum_{i=1}^{n} k_{b, i} \frac{W_{B, i} d_{t h, i}}{L_{B, i}}
$$

with

- $i$ an index to a specific layer,

- $N$ the number of support beams, typically 2 or 4 ,

- $k_{b}$ the effective thermal conductivity of the beam measured in $\mathrm{W} /(\mathrm{m} \mathrm{K})$,

- $W_{B}$ the width of the beam measured in $\mathrm{m}$,

- $d_{t h}$ the thickness of the beam measured in $\mathrm{m}$, and

- $L_{B}$ the length of the beam measured in $\mathrm{m}$.

It has also recently been proposed to include the constriction resistance that results when two thermal conducting paths of different widths are connected, which leads to a constriction effect experienced by the heat flow. This results in an additional radial thermal resistive component [13, 24, 25]. The thermal conductivity can be modified to include for the radial thermal conductive component, with the transformed thermal conductivity given by [24]

$$
k_{b}^{\prime}=\frac{\pi k_{b} L_{M}}{\pi L_{M}+2 W_{M} \ln \left(\frac{\pi W_{M}}{2 W_{B}}\right)}=\frac{k_{b}}{1+\frac{2 W_{M}}{\pi L_{M}} \ln \left(\frac{\pi W_{M}}{2 W_{B}}\right)}
$$

with $L_{M}$ and $W_{M}$ the membrane length and width respectively.

Another component that results in heat flow and contributes to $G_{e f f}$ is the atmospheric or gaseous thermal conductance, $G_{a t m}$, given as [23]

$$
G_{a t m}=k_{a i r} A_{M} / d_{\lambda}
$$

with [18, 26]

$$
k_{a i r}=\frac{k_{a i r, 0}}{1+\frac{7.6 \times 10^{-5}}{p \cdot d_{\lambda} / T_{m}}}
$$

where

- $A_{M}=L_{M} \times W_{M}$ is the area of the membrane in $\mathrm{m}^{2}$,

- $k_{a i r, 0}$ is the thermal conductivity of air at room temperature and pressure $(0.00284 \mathrm{~W} /(\mathrm{m} \mathrm{K}))$,

- $p$ is the gas pressure measure in $\mathrm{Pa}$, and

- $d_{\lambda}$ is the cavity depth measured in $\mathrm{m}$.

As is apparent from Eq. (4), the gaseous thermal conduction is calculated as a function of the surface area of the plate. This holds true only under the assumption that the entire plate region has an even temperature distribution, which intuitively one realises is not strictly true for a microbolometer. The reader is also reminded of the fact that the $\mathrm{VO}_{\mathrm{x}}$ layer is smaller than the plate area, and also has a much larger resistance compared to the $\mathrm{Ti}$ conductor over the support beams. Therefore, almost no electrical power is dissipated in the heating of the support beams since there is almost no voltage drop, unlike the case for Ti thin-film devices where the compensated area extends over a portion of the support legs [19, 20]. These considerations suggest that the conventional conduction model may not be accurate, and motivated the implementation in simulation and the manufacturing of numerous $\mathrm{VO}_{\mathrm{x}}$ device designs.

We have implement numerous simulation models with a wide range of different geometries using CoventorWare, an industry leading finite element method (FEM) MEMS multiphysics simulation platform [32]. We have consistently observed from the simulation results of the various $\mathrm{VO}_{\mathrm{x}}$ devices that a radial heat distribution pattern exists that is inconsistent with the general assumption of a uniform temperature distribution over the membrane. We have observed that the surface area that is associated with the average $\mathrm{VO}_{\mathrm{x}}$ layer temperature can be bound by an ellipse within the $\mathrm{VO}_{\mathrm{x}}$ layer, as illustrated in Fig. $2 \mathrm{~b}$ for one example, where the temperature distribution is plotted and bound by the locus of the average $\mathrm{VO}_{\mathrm{x}}$ temperature. We will show that the elliptical approximation improves the analytic prediction significantly over a wide range of device geometries. Since the $\mathrm{VO}_{\mathrm{x}}$ layer will also heat up the remaining area of the membrane, the ellipse should also be scaled. This second ellipse may be offset depending on the support beam configuration. Therefore, it is proposed that the conventional membrane area is replaced with a new elliptical surface area that fits within the borders of the $\mathrm{VO}_{\mathrm{x}}$ layer, which is then scaled by the ratio of the membrane surface area and $\mathrm{VO}_{\mathrm{x}}$ resistance surface area, as illustrated in Fig. 2a. Thus, the introduced elliptical surface area method utilises an effective area to replace $A_{M}$ in Eq. (4), given by

$$
A_{e f f}=\pi \frac{W_{R} L_{R}}{4} \frac{A_{M}}{A_{V O_{x}}}=\pi \frac{W_{M} L_{M}}{4}
$$


Compensated effective elliptical membrane area

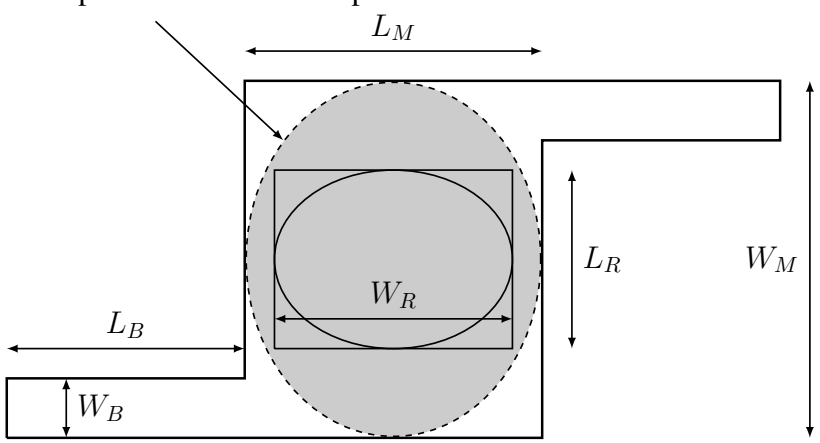

(a) A schematic of the proposed method to calculate the approximate effective membrane area of $\mathrm{VO}_{\mathrm{x}}$ microbolometers.

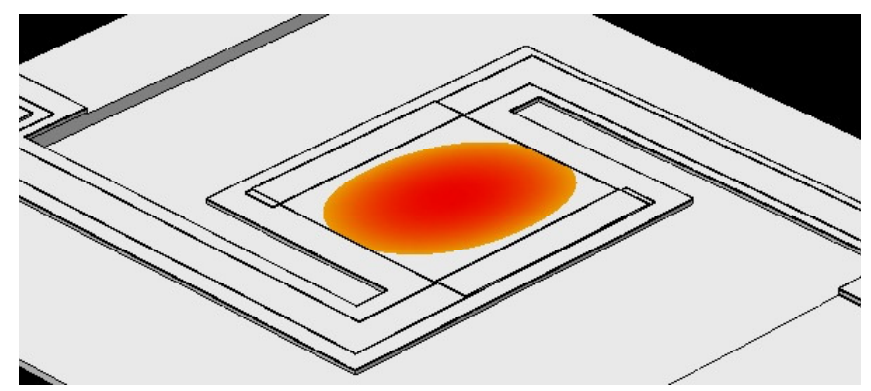

(b) A simulation model illustrating the elliptical boundary associated with the locus of the average $\mathrm{VO}_{\mathrm{x}}$ temperature.

Figure 2: The proposed improvement to the analytic gaseous thermal conduction model for $\mathrm{VO}_{\mathrm{x}}$ microbolometers by employing an elliptical surface area.

where the device dimensions are tabulated and clarified in Table 1 and illustrated in Fig. 2a. We also define $A_{V O_{x}}=$ $L_{R} \times W_{R}$, and considering a cancellation of terms, it becomes clear that the model determines the effective surface area as an ellipse bound by the membrane dimensions only.

Finally, the detector itself consists of a temperature sensitive material that changes it's electric properties proportional to the change in the temperature it experiences. This material is placed on top of the plate and will experience a temperature increase proportional to the thermal radiation and Joule heating from biasing. Therefore, it requires to be properly thermally isolated from the bulk which serves as reference temperature, $T_{0}$. The sensitivity of the material to temperature variation is referred to as the temperature coefficient of resistance (TCR), $\alpha$ and the temperature dependent resistance of the device, $R_{B}$, is typically considered over the temperature range of interest (restricted to less than $20{ }^{\circ} \mathrm{C}$ increase) as a linear relationship given by

$$
R_{B}=R_{B, 0}\left[1+\alpha\left(T_{m}-T_{0}\right)\right]
$$

with $R_{B, 0}$ the reference resistance of the device as determined at temperature $T_{0}$ typically selected as $T_{s u b}$. Intuitively, one would expect to achieve a large sensitivity if the microbolometer exhibits a large IR absorption factor, a large TCR and a low thermal conductance to the bulk and ambient.

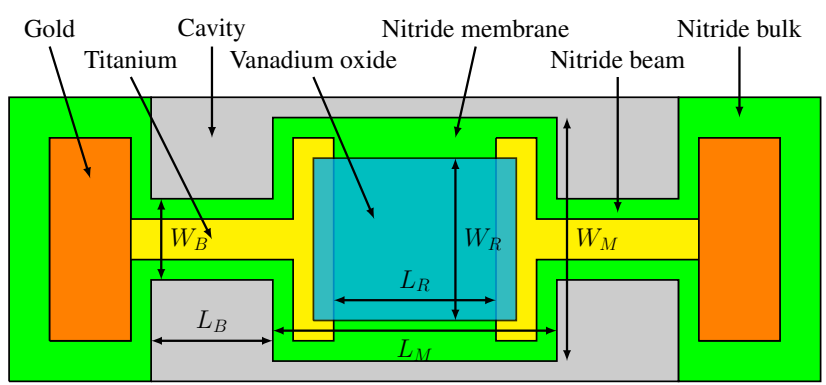

(a) Generic $\mathrm{VO}_{\mathrm{x}}$ device design with the relevant definitions of dimensions shown.

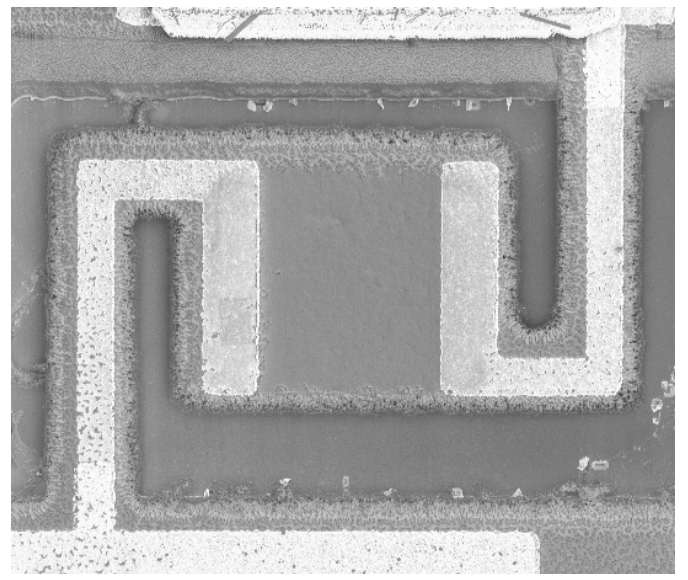

(b) A SEM image of one of the manufactured devices.

Figure 3: A generic $\mathrm{VO}_{\mathrm{x}}$ device design and manufactured device.

\subsection{Device designs}

The material properties of the different materials used to construct the microbolometer devices play a significant role in the device performance parameters. Notably, the thermal conductivity, $k_{i}$ of the materials used to construct the support beams plays a significant role in the thermal conductance, $G_{l e g}$ that will be achieved under vacuum conditions, especially that of silicon nitride in typical microbolometer designs. The various material densities, $\rho_{i}$, and specific heat, $c_{i}$, play a major role in the thermal time response, $\tau$ and the thermal capacity, $H$, of the device. The material properties of interest for this study are summarised in Table 2

Numerous different $\mathrm{VO}_{\mathrm{x}}$ device designs were manufactured to investigate the effect of each of the various different device properties, from which it is possible to characterise the parameters associated with the heat flow problem of Eq. (1). A summary of the device designs and their associated dimensions and surface characteristics are provided in Table 1. with a typical device design illustrated in Fig. 3a. The attentive reader may have noticed the curious design choice of four beams (device VOX4) which is not standard practice for microbolometer design. This device design is included to allow for a comparison with i) a design with two beams of width $16 \mu \mathrm{m}$ (VOX7), and also with ii) dual detector element device designs. 


\begin{tabular}{lcccccccccc}
\hline Device & $\begin{array}{c}W_{B} \\
{[\mu \mathrm{m}]}\end{array}$ & $\begin{array}{c}L_{B} \\
{[\mu \mathrm{m}]}\end{array}$ & $N$ & $\begin{array}{c}W_{M} \\
{[\mu \mathrm{m}]}\end{array}$ & $\begin{array}{c}L_{M} \\
{[\mu \mathrm{m}]}\end{array}$ & $\begin{array}{c}W_{R} \\
{[\mu \mathrm{m}]}\end{array}$ & $\begin{array}{c}L_{R} \\
{[\mu \mathrm{m}]}\end{array}$ & $\begin{array}{c}A_{M} \\
{\left[\mu \mathrm{m}^{2}\right]}\end{array}$ & $\begin{array}{c}A_{V O_{x}} \\
{\left[\mu \mathrm{m}^{2}\right]}\end{array}$ & $\begin{array}{c}A_{\text {eff }} \\
{\left[\mu \mathrm{m}^{2}\right]}\end{array}$ \\
\hline VOX4 & 8 & 26 & 4 & 30 & 30 & 22 & 16 & 900 & 352 & 707 \\
VOX7 & 16 & 26 & 2 & 40 & 40 & 32 & 26 & 1600 & 832 & 1257 \\
VOX15 & 12 & 26 & 2 & 40 & 40 & 32 & 26 & 1600 & 832 & 1257 \\
VOX32 & 8 & 26 & 2 & 40 & 30 & 32 & 16 & 1200 & 512 & 942 \\
VOX33 & 8 & 26 & 2 & 30 & 40 & 22 & 26 & 1200 & 572 & 942 \\
VOX36 & 10 & 71 & 2 & 40 & 40 & 30 & 26 & 1600 & 780 & 1257 \\
\hline
\end{tabular}

Table 2: Summary of the most important material properties that are used in the manufacturing of microbolometers.

\begin{tabular}{lcccc}
\hline Material & $\begin{array}{c}\text { Density } \\
{\left[\mathrm{g} / \mathrm{cm}^{3}\right]}\end{array}$ & $\begin{array}{c}\text { Specific Heat } \\
{[\mathrm{J} /(\mathrm{g} \mathrm{K})]}\end{array}$ & $\begin{array}{c}\text { Electric Conductivity } \\
{[\mathrm{S} / \mathrm{m}]}\end{array}$ & $\begin{array}{c}\text { Thermal Conductivity } \\
{[\mathrm{W} /(\mathrm{m} \mathrm{K})]}\end{array}$ \\
\hline $\mathrm{SiO}$ & $2.15-2.2[27]$ & $1.0[27]$ & $10^{-16}-10^{-14}[28,29]$ & $0.76-1.42[30]$ \\
$\mathrm{Si}_{3} \mathrm{~N}_{4}$ & $2.4-3.2[28,[29]$ & $0.69-1.73[28,17]$ & $10^{-10}$ & $1.65-5.1[30,[17]$ \\
$\mathrm{Ti}$ & $4.51[31]$ & $0.5[31]$ & $2.56 \cdot 10^{6}[32]$ & $16.3[31]$ \\
$\mathrm{VO}_{\mathrm{x}}$ & 4.57 & 1.0 & $203.6[32]$ & 15 \\
$\mathrm{Air}$ & 0.00116 & 1.007 & 0 & 0.0284 \\
\hline
\end{tabular}

\section{Methodology}

\subsection{Approach for the simulation method}

Multiphysics simulation platforms are extremely effective in simulating $3 \mathrm{D}$ device structures. This is possible by means of meshing the device structures to allow for finite points within the structure to be simulated. These simulation platforms would typically include the ability to design the necessary masks used in the manufacturing steps, as well as the processing steps required for manufacturing with the appropriate design specifications. The software can then generate the 3D models and apply a meshing algorithm. Coventorware is an industry leading multiphysics MEMS simulator and was used for the simulation portion of this work.

The essential material properties for the various materials found in the microbolometers are summarised in Table 2. The properties of air are included to enable the simulation of thermoelectric effects at atmospheric pressure. Eq. (5) allows for the modelling of various gas pressure scenarios by modifying the thermal conductivity appropriately.

The standard material properties database of the simulation software does not include the temperature dependence for the thin-film Ti nor the $\mathrm{VO}_{\mathrm{x}}$ resistor by default, but it does allow the possibility to include such functionality manually. This is done by using either a polynomial with temperature as dependent variable to calculate the appropriate electric conductivity, or to utilise a table with temperature to corresponding electric conductivity columns for a suitable mapping. Fig. 4 illustrates the implemented temperature dependence of the electric conductivity for both the $\mathrm{Ti}$ and $\mathrm{VO}_{\mathrm{x}}$ thin-film layers, where the data was extracted from experimental measurements of the manufactured test structures by considering the device resistance, number of resistive squares and the thickness of the resistor film.

A thermomechanical solver was selected to simulate electrothermal physics in the steady state. The solver can be used to simulate a variety of input scenarios applied to the meshed model, including temperature, heat flux, displacement, current density and pressure. The approach for this work is to apply an electrical stimulus in the form of an electric potential to the gold contacts of the device as a boundary condition, and then to investigate the resulting thermal effects. The selection of this choice is motivated by the fact that this approach very closely relates to the actual experimental procedure. Furthermore, the substrate is considered a thermal heat sink with constant temperature selected as $300 \mathrm{~K}$, which is applied as a fixed boundary condition. During a steady state analysis, the input potential is swept over a voltage range to investigate the corresponding temperature of the resistive element. It is possible to extract the instantaneous temperature at any point within the simulated structure, but also to calculate the average temperature of the material layer forming the electric resistance that is affected by the electric stimulus. The latter choice is preferred, since it corresponds well to the experimental method, where measurements reflect the resistance changes of the resistive element between the gold contacts.

Recall from Eq. (1) that the thermal losses may be modelled as $G_{e f f} \Delta T=\left(G_{a t m}+G_{l e g}\right)\left(T_{m}-T_{s u b}\right)$ if a purely electrical characterisation is performed and the device is also operating at room temperature where $T_{s u b} \approx T_{a}$. Once the temperature and electrical power are known, the thermal con- 


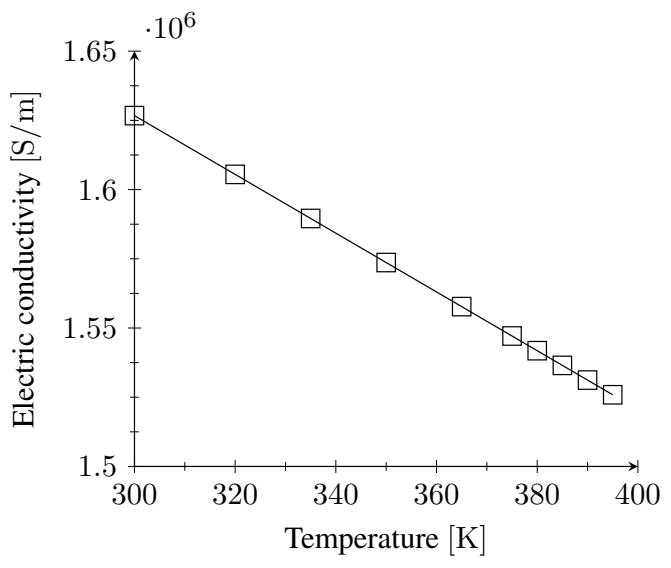

(a) Extracted electric conductivity of Ti thin film layer.

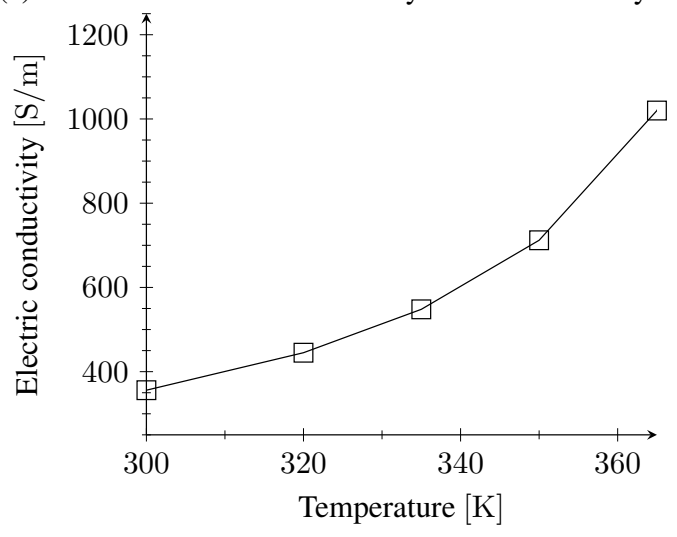

(b) Extracted electric conductivity of $\mathrm{VO}_{\mathrm{x}}$ thin film layer.

Figure 4: The thermal conductivity profiles that include the effect of temperature dependence used for the Coventorware simulations. The profiles are extracted from experimental results to compensate for manufacturing variation.

ductance may be calculated for the the simulated structure at steady state as

$$
G_{e f f}=\frac{P_{e}}{\Delta T}
$$

where $G_{e f f}$ represents the effective thermal conductance as discussed earlier.

\subsection{Approach for the experimental method}

It should be clear from Eq. (7) that direct knowledge of the TCR, $\alpha$, and the resistance, $R_{B}$, is required for successful characterisation of the microbolometer resistance. A method to measure $R_{B}$, and $\alpha$, in a controlled oven with variable temperature by means of a purely electrical method has been described [21]. The device is biased in the active region with a constant current in a static condition, without optical input power. Measuring at atmospheric pressure minimises self-heating due to the larger thermal conductivity that results from the thermal conduction through the gas [33]. A calibrated Hewlett Packard 4155B parameter analyser is used as current source and senses the device output voltage. A constant current is applied and the temperature of the oven is selected as the variable input parameter. The measured experimental output is the bolometer volt- age, from where the bolometer resistance, $R_{B}$, may be easily determined by Ohm's law, and $\alpha$ is determined from the slope of the resistance to temperature graph as

$$
\alpha=1 / R_{B, 0} \frac{\partial R_{B}\left(\bar{T}_{m}\right)}{\partial T}
$$

where $R_{B, 0}$ is determined from the I-R curve at the point where $I_{B}=0 \mathrm{~mA}$ at the reference temperature.

Once $\alpha$ and $R_{B, 0}$ are known, the effective thermal conductance, $G_{e f f}$, may be extracted from the temperature rise of the substrate due to self-heating [34] in the microbolometer element by noting that the biasing power, $I_{B}^{2} R_{B}$ is equivalent to the power dissipated by the substrate, $G_{e f f} \Delta T$. This is similar to the approach for Eq. (8). This should become clear when substituting $\Delta T=I_{B}^{2} R_{B} / G_{e f f}$ into Eq. (7p) to obtain

$$
R_{B}=R_{B, 0}(1+\alpha \Delta T)=R_{B, 0}\left(1+\alpha \frac{I_{B}^{2} R_{B}}{G_{e f f}}\right)
$$

Extraction of $G_{e f f}$ may, therefore, be done by finding the slope of a linear fitted curve of the device input power and temperature increase.

\section{Results and Discussion}

The vanadium oxide samples for this investigation are prepared in two steps. First, by means of a lift-off photo lithography, a high purity vanadium metal is deposited on the nitride membrane covered by a thin film of silicon dioxide by means of reactive ion beam sputtering to obtain the $\mathrm{VO}_{\mathrm{x}}$ thin film with a thickness of $150 \mathrm{~nm}$. This film consists of vanadium oxide compositions of $\mathrm{V}_{2} \mathrm{O}_{3}$ and $\mathrm{VO}_{2}$. This is followed by a post oxidation process that is accomplished by annealing the devices in a hydrogen atmosphere. In order to ensure that the devices are compatible with standard CMOS processes, the temperature for the preparation was $400 \pm 5{ }^{\circ} \mathrm{C}$. This typically results in a $\mathrm{VO}_{\mathrm{x}}$ sheet resistance that ranges from $10 \mathrm{k} \Omega / \square$ to $200 \mathrm{k} \Omega / \square$. The obtained sheet resistance from annealing can be increased if necessary with a hot plate at $300{ }^{\circ} \mathrm{C}$ for three minutes to oxidise the vanadium oxide. Another silicon dioxide layer of 100 $\mathrm{nm}$ is deposited as screen off oxide to ensure that the $\mathrm{VO}_{\mathrm{x}}$ is passivated.

The first step towards the characterisation of the devices is to place them in an oven with adjustable temperature that is ranged between room temperature and $80{ }^{\circ} \mathrm{C}$. It was observed that the semiconductor like devices starts to respond in a nonlinear fashion above $65{ }^{\circ} \mathrm{C}$ as reported in literature [35] when the material transitions to a metal type, and therefore the results are limited to $65^{\circ} \mathrm{C}$, which is a reasonable assumption for most operating scenarios. The TCR for the manufactured $\mathrm{VO}_{\mathrm{x}}$ prototypes are extracted and calculated to be between $-0.98 \% / \mathrm{K}$ and $-1.19 \% / \mathrm{K}$. It will be assumed to be $-1 \% / \mathrm{K}$. Furthermore, the average sheet resistance obtained for the manufacture Ti thin-film is $R_{S H}=6.17 \Omega / \square$ and for the $\mathrm{VO}_{\mathrm{x}}$ thin-film devices is $R_{S H}=31 \mathrm{k} \Omega / \square$. 
The first results presented are of simulated parametric voltage sweeps applied to the Ti device gold contacts, ranging from $0 \mathrm{~V}$ to $0.5 \mathrm{~V}$ under vacuum conditions and over a larger range from $0 \mathrm{~V}$ to $1 \mathrm{~V}$ at atmospheric pressure. The first experiment facilitates the extraction of the beam thermal conductance, $G_{l e g}$, while the second experiment allows to extract the total thermal conductance, $G_{e f f}$. The thermal conductance is extracted from the slope of the $\mathrm{P}-\mathrm{T}$ graph when considering the heat balance model of Eq. (1) and specifically Eq. (8). These results for the average $\mathrm{VO}_{\mathrm{x}}$ layer temperature and the power consumption associated with the biasing point are illustrated in Fig. 5. The beam thermal conductance is indicate in Fig. 5a for device VOX36 and the effective thermal conductance in Fig. $5 b$ for device $V O X 4$.

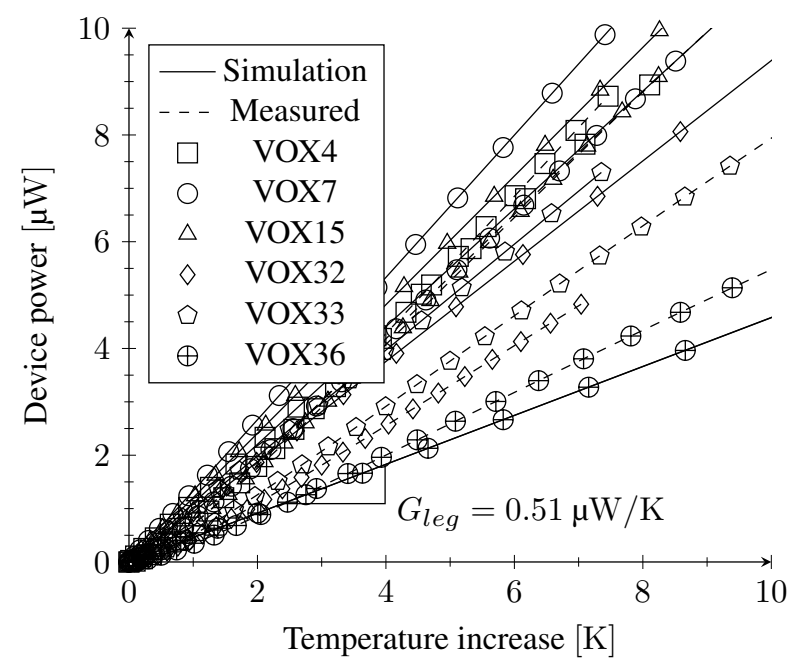

(a) Simulated temperature curves at different power levels for devices under vacuum conditions.

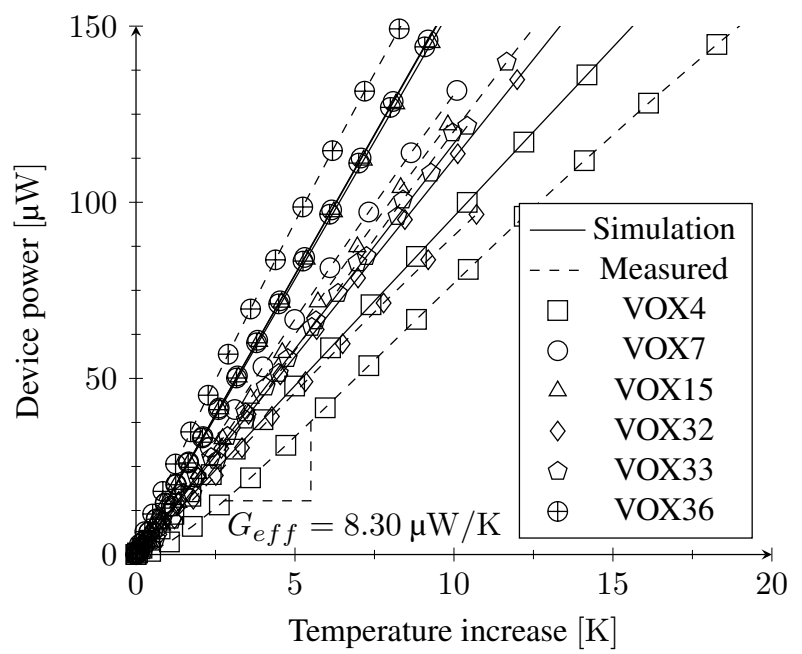

(b) Simulated temperature curves at different power levels for devices at atmospheric pressure.

Figure 5: Simulated temperature response of various $\mathrm{VO}_{\mathrm{x}}$ devices at different power levels.

Also indicated on the graphs are the results extracted from the experimental measurements. The input current is swept up
Table 3: A comparison of the thermal beam conductance, $G_{l e g}$, of the $\mathrm{VO}_{\mathrm{x}}$ devices under vacuum conditions.

\begin{tabular}{lcccc}
\hline Device & $\begin{array}{c}\text { Conventional } \\
\text { theory }^{(a)} \\
{[\mu \mathrm{W} / \mathrm{K}]}\end{array}$ & $\begin{array}{c}\text { Constriction } \\
\text { method }^{(b)} \\
{[\mu \mathrm{W} / \mathrm{K}]}\end{array}$ & $\begin{array}{c}\text { Simulated } \\
\text { results } \\
{[\mu \mathrm{W} / \mathrm{K}]}\end{array}$ & $\begin{array}{c}\text { Experimental } \\
\text { measurement } \\
{[\mu \mathrm{W} / \mathrm{K}]}\end{array}$ \\
\hline VOX4 & 2.49 & 1.26 & 1.07 & 1.20 \\
VOX6 & 1.59 & 1.08 & 1.21 & 0.94 \\
VOX7 & 1.93 & 1.22 & 1.33 & 1.13 \\
VOX8 & 2.49 & 1.46 & 1.28 & 1.22 \\
VOX13 & 0.24 & 0.22 & 0.22 & 0.48 \\
VOX15 & 1.59 & 1.08 & 1.08 & 1.13 \\
VOX23 & 0.58 & 0.50 & 0.49 & 0.48 \\
VOX24 & 1.25 & 0.92 & 0.92 & 0.78 \\
VOX32 & 1.25 & 0.95 & 0.92 & 0.71 \\
VOX33 & 1.25 & 0.90 & 0.88 & 0.82 \\
VOX36 & 0.52 & 0.45 & 0.51 & 0.57 \\
VOX39 & 0.60 & 0.52 & 0.56 & 0.50 \\
\hline
\end{tabular}

(a) Theoretical calculation using the standard approach with Eq. (2).

(b) Theoretical calculation using the compensated method by including the thermal constriction resistance with Eq. (3).

to $20 \mu \mathrm{A}$ for the devices within the evacuated dewar, while the input current of the devices at atmospheric pressure can be increased to a higher value of $100 \mu \mathrm{A}$ due to the expected increase in effective thermal conduction under atmospheric pressure. Second order fitted curves are derived from the I-V characteristics obtained using the parameter analyser and $\Delta T$ is determined using Eq. (10). Finally, $G_{\text {eff } f}$ is extracted from the slope of the $\Delta T-\mathrm{P}$ curves of Fig. 5 Once again it can be observed that electrical performance of the detectors under vacuum conditions is relatively lower than those of the devices at atmospheric pressure and this leads to a significant increase in the slope when comparing the devices under vacuum conditions with the measurements done at atmospheric pressure. This is due to the fact that the effective thermal conductance at atmospheric pressure consists of the additional gaseous thermal conductance component that is significantly larger than the beam thermal conductance. The additional thermal loss associated with the gaseous thermal conductance requires additional input power in order to compare similar temperature increases.

The various beam thermal conductance results for the $\mathrm{VO}_{\mathrm{x}}$ devices are tabulated in Table 3 , from which it can be observed that the experimental results obtained follow the expected theoretical and simulated results very closely. If one considers that the beam thermal conductance is related to the ratio of the beam width and length, by considering Eq. 22 a good linear relationship is achieved over various $\mathrm{VO}_{\mathrm{x}}$ devices with a slope of $k_{b} d_{t h}$. Finding appropriate linear fitted curves and using $k_{b}=1.65$ $\mathrm{W} /(\mathrm{m} \mathrm{K})$ as the value for the thermal conductivity for a CVD layer of silicon nitride results in a beam thickness of $d_{t h}=1.09$ $\mu \mathrm{m}$ for the theoretical case, $d_{t h}=1.11 \mu \mathrm{m}$ for the simulated data and $d_{t h}=1.02 \mu \mathrm{m}$ for the manufactured devices. It is clear that the manufactured devices measured very closely to the design value of $d_{t h}=1 \mu \mathrm{m}$. 
Table 4: A comparison of the total and effective thermal conductance, $G_{e f f}$, of the $\mathrm{VO}_{\mathrm{x}}$ devices at atmospheric pressure.

\begin{tabular}{lccccc}
\hline Device & $\begin{array}{c}\text { Conventional } \\
\text { theory }(a) \\
{[\mu \mathrm{W} / \mathrm{K}]}\end{array}$ & $\begin{array}{c}\text { Conventional } \\
(\mathrm{improved}) \\
{[\mu \mathrm{W} / \mathrm{K}]}\end{array}$ & $\begin{array}{c}\text { Elliptical } \\
\text { method }(c) \\
{[\mu \mathrm{W} / \mathrm{K}]}\end{array}$ & $\begin{array}{c}\text { Simulated } \\
\text { results } \\
{[\mu \mathrm{W} / \mathrm{K}]}\end{array}$ & $\begin{array}{c}\text { Experimental } \\
\text { measurement } \\
{[\mu \mathrm{W} / \mathrm{K}]}\end{array}$ \\
\hline VOX4 & 14.28 & 13.33 & 10.80 & 9.55 & 8.30 \\
VOX6 & 22.55 & 22.04 & 17.38 & 15.71 & 11.91 \\
VOX7 & 22.89 & 22.18 & 17.54 & 15.91 & 13.27 \\
VOX8 & 23.45 & 22.63 & 17.69 & 17.69 & 11.77 \\
VOX13 & 18.16 & 18.14 & 14.30 & 14.39 & 13.45 \\
VOX15 & 22.55 & 22.04 & 17.54 & 15.65 & 12.74 \\
VOX23 & 21.54 & 21.46 & 16.96 & 15.62 & 9.27 \\
VOX24 & 22.21 & 21.88 & 17.38 & 15.44 & 10.88 \\
VOX32 & 16.97 & 16.67 & 13.29 & 11.41 & 9.31 \\
VOX33 & 16.97 & 16.62 & 13.25 & 11.67 & 12.26 \\
VOX36 & 21.48 & 21.41 & 16.91 & 16.51 & 15.42 \\
VOX39 & 12.39 & 12.31 & 9.78 & 9.55 & 10.49 \\
\hline
\end{tabular}

(a) Theoretical calculation using the standard approach with Eq. (4) and Eq. (2).

(b) Theoretical calculation using the standard approach with Eq. (4), but including the thermal constriction resistance with Eq. (3).

(c) Theoretical calculation using the compensated method by including the thermal constriction resistance with Eq. (3) and employing the elliptical method of Eq. (6).

It should be clear from Table 4 that the theoretical results calculated with the conventional analytic method deviate from the simulation results by a substantial amount. As can be seen from the tabulated data, the elliptical model dramatically improves the theoretical results, although there is still some inaccuracies in the estimation of the effective area dependent on the device geometry. It is also evident that the experimental data resulted in values smaller than anticipated. It was suspected that the membranes are bending outwards altering the value of $d_{\lambda}$, and a microscopic study of the devices was performed with a confocal microscope using profilometry to confirm the suspicion. The measured membrane to substrate gap ranged between $2.2 \mu \mathrm{m}$ and $2.7 \mu \mathrm{m}$ for various devices and is larger than the design value of $2 \mu \mathrm{m}$.

To investigate the severity of this phenomenon further, the total thermal conductance obtained at atmospheric pressure is plotted against the membrane area, as the slope of the graph will then represent $k_{a i r} / d_{\lambda}$ considering Eq. (4). This is illustrated in Fig. 6 where the total thermal conductance is plotted against the membrane area, with the assumption that the beam thermal conductance component is small enough not to influence the result significantly. For the ideal theoretical case,

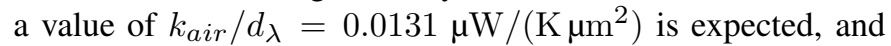
is obtained almost exactly as can be observed from the figure. This provides support for the assumption that the influence of $G_{l e g}$ may be disregarded. A slight offset from zero is observed and expected, considering the role of the $G_{l e g}$ with reference to Eq. (1) in the overall thermal conductance. The offset is comparable to the average values of the results in Table 3 .

It is assumed that the simulated results will provide the most

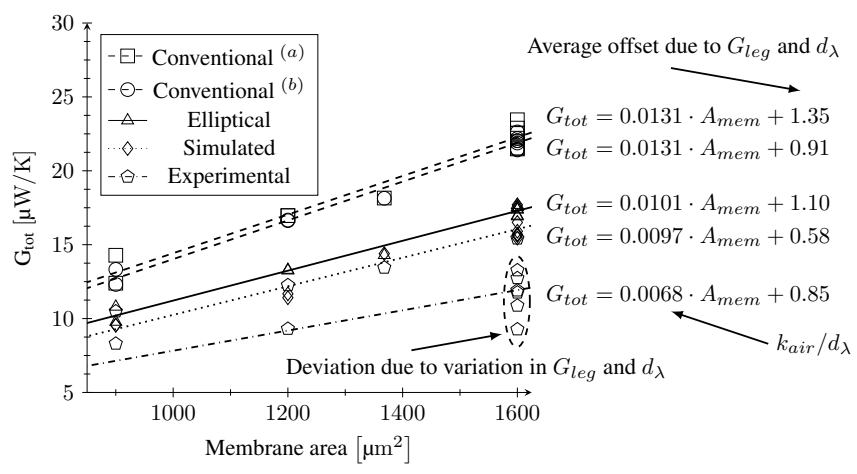

Figure 6: A comparison of the thermal conductance components using the standard conventional analytic model (a), the standard method with compensation for the restriction resistance (b), the proposed elliptical method, the extracted simulated and the extracted experimental plotted against the membrane area for various $\mathrm{VO}_{\mathrm{x}}$ devices at atmospheric pressure. A linear fitted curve for each data set is also plotted.

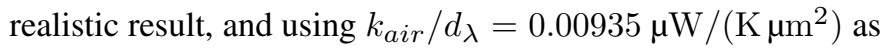
a reference, it is observed that the conventional method overestimates the thermal conductance for $\mathrm{VO}_{\mathrm{x}}$ based microbolometers on average by approximately $40 \%$, a significant error. Since the gaseous thermal conduction dominates the effective thermal conduction, the effect of including the constriction resistance in the calculation is limited and the conventional curves plotted in Fig. 6 are close to one another. The proposed analytic model employing an area scaling mechanism to determine an effective thermal area, however, reduces this overestimation by a large margin to roughly $8 \%$ for the designed devices. It can also be observed that the experimentally obtained result is below that of the reference simulation result by approximately $16 \%$ on average, and using this to scale the value of $d_{\lambda, \text { sim }}=2 \mu \mathrm{m}$, an average value of $d_{\lambda, \exp }=2.39 \mu \mathrm{m}$ is obtained. This value falls within the range observed with profilometry.

One last point worth discussion is the observation from Fig. 6 that, taking for example all the devices with $A_{M}=1600$ $\mu \mathrm{m}^{2}$, the thermal conductance results obtained differ somewhat between the various devices. Recall that Eq. (4) will provide a constant value for constant $A_{M}$, therefore the differences and resulting deviation are attributed to the various $G_{l e g}$ values between devices. However, this is not the only cause of the deviation. It was observed during the profilometry experiments that there is some deviation between devices with regards to $d_{\lambda, \text { exp }}$, which will also influence $G_{\text {eff }}$.

\section{Conclusions}

A number of conclusions can be drawn from the presented work. It has come to light that the conventional analytic method for calculating the atmospheric thermal conductance component of uncooled $\mathrm{VO}_{\mathrm{x}}$ microbolometers grossly overestimates the actual value due to an inaccurate assumption based on a uniform temperature distribution of the device plate. This is supported by both simulation and experimentally obtained results. It is clear that the proposed method to calculate the thermal conductance component by using the effective thermal area 
dramatically improves the analytic result, reducing the average error from $40 \%$ to $8 \%$. The marginal error that remains is due to the fact that the elliptical method is suggested as an approximate solution that is rooted in the observation of numerous simulated $\mathrm{VO}_{\mathrm{x}}$ designs, hence it has not been derived from first principles. This will require solving a two dimensional heat flow problem that is considered for future research, although it is very likely to offer a much more complex solution. It should be emphasised that the elegance of the elliptical method lies in the simplicity of its application to microbolometer designers, that may not necessarily wish to spend a large computational effort on a very complex analytic model. It is also evident that the plate and substrate separation distance plays a significant role when comparing experimentally obtained results with those of simulation or analysis. The role of the internal stresses between the material layers of MEMS structures have not been well quantified in literature, and analytic methods could also benefit from future investigation.

\section{Acknowledgements}

The authors thank the Advanced Manufacturing Technology Strategy (AMTS) of the Department of Science and Technology, South Africa, for the financial support of the research, as well as the National Research Foundation (NRF) for Sabbatical Grants to Complete Doctoral Degrees Funding Instrument (UID Number 86451).

\section{References}

[1] K. C. Liddiard, Novel concepts for low-cost IR security sensors, in: Defense and Security, International Society for Optics and Photonics, 2005, pp. 693-702.

[2] A. Kosarev, A. Torres, M. Moreno, R. Ambrosio, Thin film uncooled microbolometers based on plasma deposited materials, Canadian Journal of Physics 92 (7/8) (2014) 570-575.

[3] T. W. Parker, C. A. Marshall, M. Kohin, R. Murphy, Uncooled infrared sensors for surveillance and law enforcement applications in: Surveillance and Assessment Technologies for Law Enforcement, Vol. 2935, 1997, pp. 182-187. doi:10.1117/12.266799

URL http://dx.doi.org/10.1117/12.266799

[4] M. A. Gallo, D. S. Willits, R. A. Lubke, E. C. Thiede, Low-cost uncooled IR sensor for battlefield surveillance in: Infrared Technology XIX, Vol. 2020, 1993, pp. 351-362. doi:10.1117/12.160556

URL http://dx.doi.org/10.1117/12.160556

[5] K. C. Liddiard, Application of mosaic pixel microbolometer technology to very high-performance, low-cost thermography and pedestrian detection in: Infrared Technology and Applications XXXIX, Vol. 8704, 2013, pp. 87043F-87043F-9. doi:10.1117/12.2018593

URL http://dx.doi.org/10.1117/12.2018593

[6] J.-A. Mellish, A. Hindle, J. Skinner, M. Horning, Heat loss in air of an Antarctic marine mammal, the Weddell seal Journal of Comparative Physiology B 185 (1) (2015) 143-152. doi:10.1007/ S00360-014-0868-2 URL http: //dx.doi.org/10.1007/s00360-014-0868-2

[7] C. L. Herry, M. Frize, E. Bariciak, Assessment of abdominal skin temperature change in premature newborns with NEC compared to healthy controls, in: 5th European Conference of the International Federation for Medical and Biological Engineering, Springer, 2012, pp. 191-194.

[8] G. Ntonfo, M. Frize, E. Bariciak, Detection of Necrotizing Enterocolitis in newborns using abdominal thermal signature analysis, in: 2015 IEEE International Symposium on Medical Measurements and Applications (MeMeA), IEEE, 2015, pp. 36-39.
[9] E. V. Druzhkin, T. N. Khatsevich, N. V. Brovka, Thermal viewer for medical purposes. Journal of Optical Technology 82 (7) (2015) 408-410. doi:10.1364/JOT.82.000408

URL http://jot.osa.org/abstract.cfm?URI= jot $-82-7-408$

[10] J. Huamyun, A. Malik, Multispectral and thermal images for acne vulgaris classification, in: National Postgraduate Conference (NPC), 2011, 2011, pp. 1-4. doi:10.1109/NatPC.2011.6136336

[11] E. F. J. Ring, K. Ammer, Infrared thermal imaging in medicine Physiological Measurement 33 (3) (2012) R33.

URL http://stacks.iop.org/0967-3334/33/i=3/a=R33

[12] B. Xiao, T. Dong, E. Halvorsen, Z. Yang, Y. Zhang, N. Hoivik, D. Gu, N. Tran, H. Jakobsen, Integrated micro Pirani gauge based hermetical package monitoring for uncooled $\mathrm{VO}_{\mathrm{x}}$ bolometer FPAs. Microsystem Technologies 17 (1) (2011) 115-125. doi:10.1007/ s00542-010-1186-6

URL http://dx.doi.org/10.1007/s00542-010-1186-6

[13] N. Topaloglu, P. M. Nieva, M. Yavuz, J. P. Huissoon, Modeling of thermal conductance in an uncooled microbolometer pixel Sensors and Actuators A: Physical 157 (2) (2010) 235 - 245. doi : http://dx.doi.org/ $10.1016 / j . s n a .2009 .11 .018$ URLhttp://www.sciencedirect.com/science/article/ pii/S0924424709005056

[14] W.-B. Song, J. J. Talghader, Design and characterization of adaptive microbolometers, Journal of Micromechanics and Microengineering 16 (5) (2006) 1073.

[15] N. Topaloglu, P. M. Nieva, M. Yavuz, J. P. Huissoon, A pixel-bypixel thermal conductance tuning mechanism for uncooled microbolometers, in: Microsystems and Nanoelectronics Research Conference, 2008. MNRC 2008. 1st, IEEE, 2008, pp. 201-204.

[16] H. Jerominek, F. Picard, D. Vincent, Vanadium oxide films for optical switching and detection Optical Engineering 32 (9) (1993) 2092-2099. doi:10.1117/12.143951 URL http://dx.doi.org/10.1117/12.143951

[17] P. Eriksson, J. Andersson, G. Stemme, Thermal characterization of surface-micromachined silicon nitride membranes for thermal infrared detectors, Journal of Microelectromechanical Systems 6 (1) (1997) 5561. doi:10.1109/84.557531

[18] J. Schoeman, Employing a microbolometer as a low pressure sensor in: Proc. SPIE 9257, Sensors, MEMS and Electro-Optical Systems, Vol. 9257, 2014. doi:10.1117/12.2066287 URL http://dx.doi.org/10.1117/12.2066287

[19] M. Almasri, B. Xu, J. Castracane, Amorphous silicon two-color microbolometer for uncooled IR detection. IEEE Sensors Journal 6 (2) (2006) 293-300. doi:10.1109/JSEN.2006.870139

URL http://www.scopus.com/inward/record.url? eid=2-s2.0-33645163068\&partner $I D=40 \& \mathrm{md} 5=$ 5 f0ded04d876c875e8c7ff39f717d58a

[20] M. du Plessis, J. Schoeman, W. Maclean, C. Schutte, The electro-thermal properties of integrated circuit microbolometers, SAIEE Africa Research Journal 102 (2) (2011) 40-48.

[21] J.-S. Shie, Y.-M. Chen, M. Ou-Yang, B. C. S. Chou, Characterization and modeling of metal-film microbolometer, Journal of Mircoelectromechanical Systems 5 (4) (1996) 298-306.

[22] M. Galeazzi, D. McCammon, Microcalorimeter and bolometer model, Journal of Applied Physics 93 (8) (2003) 4856-4869.

[23] F. Niklaus, C. Jansson, A. Decharat, J.-E. Källhammer, H. Pettersson, G. Stemme, Uncooled infrared bolometer arrays operating in a low to medium vacuum atmosphere: performance model and tradeoffs, in: Defense and Security Symposium, International Society for Optics and Photonics, 2007, pp. 65421M-65421M.

[24] N. Topaloglu, P. Nieva, M. Yavuz, J. Huissoon, A novel method for estimating the thermal conductance of uncooled microbolometer pixels, in: IEEE International Symposium on Industrial Electronics, ISIE 2007, 2007, pp. $1554-1558$. doi:10.1109/ISIE.2007.4374834

[25] W. M. Rohsenow, J. P. Hartnett, Y. I. Cho, et al., Handbook of heat transfer, Vol. 3, McGraw-Hill New York, 1998.

[26] J. Potkay, G. Lambertus, R. Sacks, K. Wise, A low-power pressure- and temperature-programmable micro gas chromatography column, Journal of Microelectromechanical Systems 16 (5) (2007) 1071-1079. doi: 10 . $1109 /$ JMEMS.2007.902470 
[27] 6.777J/2.751J Material Property Database

URL http://www.mit.edu/ 6.777/matprops/sio2.htm

[28] M. Madou, Fundamentals of Microfabrication: The Science of Miniaturization, Second Edition Taylor \& Francis, 2002.

URL

[29] T.-R. Hsu, MEMS and Microsystems: design, manufacture, and nanoscale engineering, 2nd Edition, John Wiley \& Sons, 2008.

[30] M. Von Arx, O. Paul, H. Baltes, Process-dependent thin-film thermal conductivities for thermal CMOS MEMS, Journal of Microelectromechanical Systems 9 (1) (2000) 136-145.

[31] 6.777J/2.751J Material Property Database

URL/http://www.mit.edu/ 6.777/matprops/titanium2. htm

[32] http://www.coventor.com/mems-solutions/products/coventorware/.

[33] K. A. Hay, D. van Deusen, T. Y. Liu, W. A. Kleinhans, Uncooled focal plane array detector development at InfraredVision Technology Corporation, in: Proceedings of SPIE: Infrared Technology and Applications XXIX, Vol. 5074, 2003, pp. 491-499.

[34] P. Eriksson, J. Y. Andersson, G. Stemme, Thermal characterization of surface-micromachined silicon nitride membranes for thermal infrared detectors, Journal of Microelectromechanical Systems 6 (1) (1997) 5561.

[35] H. Wang, X. Yi, S. Chen, Low temperature fabrication of vanadium oxide films for uncooled bolometric detectors, Infrared Physics and Technology 47 (3) (2006) 273-277. doi:10.1016/j.infrared.2005.04. 001 